\title{
Shifts in human skin and nares microbiota of healthy children and adults
}

Julia Oh', Sean Conlan', Eric C Polley ${ }^{2}$, Julia A Segre ${ }^{1 *+}$ and Heidi H Kong ${ }^{3^{*+}}$

\begin{abstract}
Background: Characterization of the topographical and temporal diversity of the microbial collective (microbiome) hosted by healthy human skin established a reference for studying disease-causing microbiomes. Physiologic changes occur in the skin as humans mature from infancy to adulthood. Thus, characterizations of adult microbiomes might have limitations when considering pediatric disorders such as atopic dermatitis (AD) or issues such as sites of microbial carriage. The objective of this study was to determine if microbial communities at several body sites in children differed significantly from adults.
\end{abstract}

Methods: Using 16S-rRNA gene sequencing technology, we characterized and compared the bacterial communities of four body sites in relation to Tanner stage of human development. Body sites sampled included skin sites characteristically involved in AD (antecubital/popliteal fossae), a control skin site (volar forearm), and the nares. Twenty-eight healthy individuals aged from 2 to 40 years were evaluated at the outpatient dermatology clinic in the National Institutes of Health's Clinical Center. Exclusion criteria included the use of systemic antibiotics within 6 months, current/prior chronic skin disorders, asthma, allergic rhinitis, or other chronic medical conditions.

Results: Bacterial communities in the nares of children (Tanner developmental stage 1) differed strikingly from adults (Tanner developmental stage 5). Firmicutes (Streptococcaceae), Bacteroidetes, and Proteobacteria ( $\beta, \gamma$ ) were overrepresented in Tanner 1 compared to Tanner 5 individuals, where Corynebacteriaceae and Propionibacteriaceae predominated. While bacterial communities were significantly different between the two groups in all sites, the most marked microbial shifts were observed in the nares, a site that can harbor pathogenic species, including Staphylococcus aureus and Streptococcus pneumonia.

Conclusions: Significant shifts in the microbiota associated with progressive sexual maturation as measured by Tanner staging suggest that puberty-dependent shifts in the skin and nares microbiomes may have significant implications regarding prevention and treatment of pediatric disorders involving microbial pathogens and colonization.

\section{Background}

Studies of microbial diseases typically focus on individual pathogens as causative agents. Recent insights into the microbial communities inhabiting the human body suggest that pathogenicity can be enhanced or impaired by shifts in bacterial communities and that disturbance in the microbial ecosystem (dysbiosis) can contribute to disease [1]. Genomic sequencing surveys of the normal

\footnotetext{
* Correspondence: jsegre@nhgri.nih.gov; konghe@mail.nih.gov

+ Contributed equally

${ }^{1}$ Genetics and Molecular Biology Branch, National Human Genome Research Institute, NIH, 49 Convent Dr., Bethesda, MD 20814, USA

${ }^{3}$ Dermatology Branch, Center for Cancer Research, National Cancer Institute,

NIH, 10 Center Dr., Bethesda, MD 20814, USA

Full list of author information is available at the end of the article
}

healthy human skin microbiome demonstrated vast complexity and diversity $[2,3]$ influenced by both environmental and host factors. The influence of sexual maturation on the human skin microbiome is not well studied, but may have a profound effect on disease predilection and outcome.

Maturation-dependent shifts in the skin microbiome may be relevant to the prevention, diagnosis, and treatment strategies for disorders such as atopic dermatitis (AD) that differ in incidence and severity as individuals physiologically mature. Prior studies have characterized

\section{() Biomed Central}

(c) 2012 Oh et al.; licensee BioMed Central Ltd. This is an open access article distributed under the terms of the Creative Commons Attribution License (http://creativecommons.org/licenses/by/2.0), which permits unrestricted use, distribution, and reproduction in any medium, provided the original work is properly cited. 
primarily cultivatable microbes $[4,5]$ on the skin of subjects of different ages, but cultivation techniques have limited capacity to characterize fastidious or uncultivable organisms, which may represent upwards of $99 \%$ of bacteria [6]. In contrast, genomic studies, including metagenomic or 16S-ribosomal RNA sequence analysis, greatly increase the range of detectable microbes. The 16S-rRNA gene is present in all bacteria/archaea and contains both variable regions, which enable taxonomic classification, and conserved regions, which serve as universal binding sites for PCR primers. Such methods have been used to investigate the healthy adult $[2,3]$ and the neonatal skin microbiomes $[7,8]$, but differences in the composition and complexity of the skin microbiome as a result of major developmental stages such as puberty have not been explored.

Progression from infancy to adulthood encompasses major biological changes with significant physiologic and endocrinologic skin alterations that, in turn, likely influence the host-microbiome relationship. Since chronological age does not necessarily correspond with a defined stage in sexual maturation $[9,10]$, use of Tanner staging of sexual maturity can provide a phenotypic assessment of the physiologic age of an individual. This study describes dramatic differences in the nares and skin microbiomes that occur in younger children (Tanner stage 1) versus adults (Tanner stage 5), with a smaller number of children in intermediate Tanner groups (stages 2 to 4 ) suggestive of a microbial shift occuring around puberty. Researchers should consider these physiologic differentials when designing and conducting investigations and developing interventions that promote healthy microbiomes.

\section{Materials and methods Subjects}

Subjects were 28 healthy individuals (no systemic antibiotics within 6 months, no current or prior chronic skin disorders, chronic medical conditions, asthma, or allergic rhinitis, ascertained via an International Study of Asthma and Allergies in Childhood questionnaire [11]) ranging in age from 2 to 40 years (Table S1 in Additional file 1). Data were analyzed retrospectively from our previous skin survey of healthy adults [2] with the addition of two samples. Children (aged 2 to 17 years) were recruited into a prospective longitudinal study approved by the institutional review board of NHGRI, NIH for the study of children with AD and healthy age-matched controls [12] with written informed consent obtained from the parents or guardians of all participating minors. All subjects were recruited from the local Washington, DC metropolitan region. The Tanner staging of puberty $[13,14]$ was determined by a board-certified physician (HHK). The study conformed to the ethical principles of the Declaration of Helsinki.

\section{Culture collection}

Nasal Staphylococcus aureus cultures were performed by the Department of Laboratory Medicine (DLM), Clinical Center, NIH. Cultures were obtained with swabs (BBLtm CultureSwab ${ }^{\mathrm{TM}}$, made by Copan for Becton, Dickinson and Company, Sparks, MD, USA) and plated on three different growth media: chocolate agar, trypticase soy agar with 5\% sheep blood, and mannitol salt agar (Remel Products, Lenexa, KS, USA). After overnight incubation at $37^{\circ} \mathrm{C}$, colonies were counted and assessed macroscopically and also microscopically following staining by Gram's stain. Identification was confirmed using Staphaurex agglutination reagent (Remel Products) or standard coagulase test. Culture results were returned as an assignment to one of four categorical levels based on number of colonies per sample: 0 (zero), 1 (scant), 2 (light), 3 (moderate), or 4 (heavy), corresponding to $0,1,2$ to 10,11 to 40 , or $>40$ colonies on a culture plate.

\section{Sample collection and processing}

All samples were collected (HHK) as described $[2,12]$. Briefly, skin preparation instructions included avoidance of bathing for 24 hours, emollients, antimicrobial soaps, or shampoos for 7 days prior to all sampling. Sampling sites included the nares $(\mathrm{N})$, antecubital fossa (Af), volar forearm (Vf), and popliteal fossa (Pf). From a $4 \mathrm{~cm}^{2}$ area, bacterial swabs (via Epicentre swabs) and scrapes (via sterile disposable surgical blade) were obtained and incubated in enzymatic lysis buffer and lysozyme for 30 minutes at $37^{\circ}$ C. For the nares, only swab samples were taken. Scrapes and swabs were obtained from the other sites as available. Correlation analysis of log proportions of genera identified in scrape versus swab sampling confirmed the similarity of techniques $\left(\mathrm{R}=0.87, P<2.2 \times 10^{-16}\right.$, data not shown).

\section{Preparation of samples for Sanger sequencing}

For the Tanner stages 1 to 4 samples, genomic DNA was extracted with slight modifications to the original protocol used for the Tanner 5 subject samples [2,12], including a change in the enzymatic lysis buffer (Epicentre MPY80200) and lysozyme (Epicentre R1802M), increase in the frequency and the duration of bead-beating $(30 \mathrm{~Hz}$, 2 minutes), higher heating temperature of the sample $\left(65^{\circ} \mathrm{C}\right)$, purification with Invitrogen columns, and reduced dilution of the eluate (to $35 \mu \mathrm{l}$ ). Full-length 16S-rRNA genes from genomic extractions were PCR amplified using the primer set $8 \mathrm{~F}$ and $1391 \mathrm{R}$, purified, cloned, and sequenced at the National Institutes of Health Intramural Sequencing Center (NISC) as described; 300 to 400 unique sequences were obtained from $16 \mathrm{~S}$ amplicons of each clinical sample. To ensure that bias was not introduced by protocol modifications between the two studies, we validated our results on a subset of samples prepared identically and sequenced via both Sanger and 454 sequencing. 
The results strongly corroborated the results presented here (data not shown).

\section{Sequence analysis pipeline}

Mothur v.1.21.0 [15] was used for sequence processing, definition of operational taxonomic units (OTUs), and downstream analyses. Sequence assembly, filtering, and alignment were performed as described [2,12]. Briefly, sequences matching the human genome were removed (Evalue $<0.1)$ then aligned using the Greengenes [16] NAST [17] aligner. Sequences were then chimera checked using the mothur implementation of UCHIME [18]. Sequences were classified using a Ribosomal Database Project naïve Bayesian classifier [19].

All uncorrected pairwise distances were calculated and OTUs defined at $97 \%$ similarity using average neighbor joining. No lane masking was applied. To estimate sampling saturation, rarefaction curves were generated for each site. Alpha diversity (community evenness and richness: Shannon diversity index, 'diversity'), and beta diversity (shared community structure/membership: theta, $\theta$ 'similarity' index) were calculated at a $97 \%$ similarity cutoff in mothur. To corroborate our OTU-based results, we also calculated phylogenetic trees using the clearcut implementation in mothur [20] (data not shown).

\section{Statistics}

All data are represented as mean \pm standard error of the mean unless otherwise indicated. Unless otherwise indicated, $P$-values were adjusted for multiple comparisons $>6$ using the p.adjust function in R using method=' $\mathrm{fdr}$ ' [21]. Statistical significance was ascribed to an alpha level of the adjusted $P$-values $\leq 0.05$; adjusted values $>0.05$ and $\leq 0.1$ were deemed to approach significance. Each site was treated as a separate dataset based on spatial physiological differences between different body niches [2]. All persample calculations are available upon request as a separate dataset. In general, analyses based on age rather than Tanner staging as a predictor variable were concordant for major taxa (data not shown).

For subjects with multiple measurements (for example, symmetric sites or multiple sampling timepoints), samples were processed independently and then pooled. Processed sequences were then subsampled at the smallest group size for that site (that is, number of Af sequences subsampled $=355, \mathrm{~N}=328, \mathrm{Pf}=317, \mathrm{Vf}=321$ and diversity statistics and proportions were calculated from the subsampled data. For alpha and beta diversity statistics, pools were subsampled $100 \times$ and resultant values averaged. Greater than 5 subsamplings yielded a $99.8 \%$ correlation with results from one subsampling, and 5 to 100 subsamplings yielded up to $99.99 \%$ similarity in value. Thus, for taxonomy-based statistics, we subsampled once. Similar results were obtained by averaging symmetric sites and using a mixed effects model with a random intercept for multiple timepoints (data not shown). Analysis of variance (ANOVA) and analysis of molecular variance (AMOVA) [22] were used with $\theta$ to determine statistically significant differences between microbial populations at different Tanner stages.

For linear regression analysis of alpha diversity of the subsampled data, Tanner stage was used as a five-level factor variable. For all subsequent analyses, we performed two sets of comparisons. First, we performed comparisons between Tanner stages 1 and 5 , which comprised the largest sample sizes. Second, we generated statistics for grouped Tanner stages 1 to 3 ('Tanner1-3') and Tanner stages 4 to 5 ('Tanner4-5') due to strong segregation of Tanner stages 2 and 3 with Tanner stage 1, and Tanner stage 4 with 5 during principal coordinates analyses. For beta diversity metrics, means and ANOVA were performed on the subsampled data and Tukey's honest significance test was used for all post hoc comparisons. To analyze the over/underrepresentation of taxonomies between different Tanner stage groups, we used a linear model using Tanner staging as a two-level predictor variable and the subsampled proportions as the dependent variable. We performed this regression on the proportions obtained using i) 12 major phyla/family categories, ii) OTUs classified at the genus level, and iii) species (Staphylococcus and Streptococcus only, for which we used custom scripts and databases to speciate).

To determine if the relative abundance of $S$. aureus in the nares was correlated with the relative abundance of $S$. aureus at other skin sites, we calculated the Spearman correlation coefficients between the relative abundances of the nares and the skin sites. To determine if presence or absence of $S$. aureus in the nares was consistent with the presence or absence of $S$. aureus $\geq 1$ skin site, we performed a Pearson's Chi-squared test for count data.

\section{Accession numbers}

Sequence data have been deposited in GenBank under the accession numbers [GQ000001] to [GQ116391] and can be accessed through BioProject ID 46333.

\section{Results}

To determine if microbial communities in children differed significantly from adults, we analyzed a total of 114,410 full-length 16S-rRNA sequences for 4 skin/nares sites in 28 individuals. Rarefaction curves indicated that our sampling provided sufficient coverage to analyze dominant members of the bacterial communities (Figure S1 in Additional file 2). Because of our interest in the role of the skin microbiome in diseases such as $\mathrm{AD}$, we analyzed data obtained from symmetric skin sites relevant to disease predilection: the antecubital fossae (Af) and popliteal fossae (Pf), the volar forearm (Vf, a control for these two sites), 
and the nares $(\mathrm{N})$, a potential reservoir for pathogens such as $S$. aureus and S. pneumoniae [23].

\section{Comparisons between Tanner stages}

Tanner staging is a standardized measurement of assessing pubertal development based on physiological characteristics, specifically primary and secondary external sex characteristics $[13,14]$. Due to natural variability in sexual maturation rates, Tanner stages are considered more accurate assessments of maturation than chronological ages.

To assess whether there is a shift in the microbial composition of children (Tanner stage 1 ) versus adults (Tanner stage 5), we calculated the variation between individuals within and between the two groups. $\theta$ index is a measure of overall microbial community similarity with values between 0 and 1: a value of 1 implies identical community structure, and a value of 0 implies dissimilar community structures.

We performed principal coordinates analysis (PCoA) based on the $\theta$ 'similarity' index, where a shorter distance between points indicates increasing similarity (Figure 1a; Figure S2 in Additional file 2). Biplot arrows show the most significant taxa contributing to axis variation; these taxa overlapped between the three skin sites and nares (Table S2 in Additional file 1). The nares microbiome at Tanner stages 1, 2, and 3 strongly segregated from the nares microbiome of Tanner stages 4 and 5 , forming two distinct clusters with significantly different centroids (AMOVA for the two centroids, $P<0.001$ ). This separation was less marked but statistically significant in the three skin sites (AMOVA $P<0.001$; Figure S2 in Additional file 2).

Since sample sizes for intermediate Tanner stages $(2,3$, and 4) were small, we assessed community differences between the stages with the largest numbers of subjects (Tanner stage 1 versus 5). To investigate trends that might occur during the physiological transition from childhood to adulthood, we also examined all of the subjects separated into two groups: Tanner stages 1 to 3 ('Tanner1-3') and Tanner stages 4 to 5 ('Tanner4-5') based on the strong segregation of Tanner stages 2 and 3 with Tanner stage 1 , and Tanner stages 4 with 5 observed in the PCoA analysis. We reported both analyses because the initial analysis of the two largest cohorts demonstrated significant differences between Tanner stages 1 (young children) and 5 (adults), and the latter analysis included a smaller number of individuals in intermediate Tanner stages, suggesting that a shift occurs in the skin and nares microbiome during the physiological transition from childhood to adulthood.

Within the nares, the interpersonal variation between children was $\theta=0.269 \pm 0.035$ and between adults was $\theta=$ $0.585 \pm 0.023$ (Figure 1b). In contrast, the mean similarity between children and adults was only $\theta=0.025 \pm 0.003$
(Figure 1b), demonstrating significantly greater similarity within the groups than between the two groups. More generally, the mean $\theta$ similarity of the Tanner 1 microbial communities differed significantly from that of the Tanner 5 microbial communities at the different skin sites (Figure 1b), exceeding baseline levels of interpersonal variation (Table S3 in Additional file 1; ANOVA $2.2 \times 10^{-16}$ $<P<8.1 \times 10^{-3}$; Af Tanner 5 versus interpersonal variation not significant). Interestingly, interpersonal variation between Tanner 5 individuals was lower than Tanner 1 individuals in the nares $\left(P<2.2 \times 10^{-16}\right)$. These trends were correspondingly significant in 'Tanner1-3' and 'Tanner4-5' groups. Furthermore, the observation that microbial 'diversity' of the nares microbiome decreased with increasing sexual maturity $\left(P=7.8 \times 10^{-03}\right.$; Figure 2; Table S4 in Additional file 1) suggests a stabilization and convergence of the nares microbiome in more mature individuals. Finally, we verified that these microbial community differences were not caused by gender-related differences; $\theta$ 'similarity' between males and females at all sites did not differ significantly regardless of Tanner stage $(P>0.05$; Table S3 in Additional file 1).

\section{Bacterial taxonomies significantly associated with Tanner stage}

To identify bacteria distinguishing Tanner 1 from Tanner 5 microbiomes, we classified the taxonomy of the sequence data; proportions of major taxa are shown in Figure 3a and Figure S3 in Additional file 2. We compared relative abundances of each bacteria at several taxonomic levels to determine differences between Tanner stages 1 and 5 at: i) a high phylum-family level; ii) a more specific genus level; and then where possible, at iii) the species level to determine the prevalence of opportunistic pathogens such as $S$. aureus and S. pneumoniae. Results comparing 'Tanner1-3' and 'Tanner4-5' groups, which were generally highly concordant with Tanner stages 1 versus 5 comparisons, are also reported in Tables S5 to S8 in Additional file 1.

\section{High-level analysis (phylum-family)}

The nares microbiomes in Tanner stage 1 individuals were primarily composed of Streptococcaceae and other Firmicutes, Bacteroidetes, and $\beta$ - and $\gamma$-Proteobacteria, while the nares microbiomes in Tanner stage 5 were strikingly dominated by Corynebacteriaceae and Propionibacteriaceae (Figure 3b, Table 1). This pattern of differential dominant phyla identified in younger children and adults was generally consistent across the three skin sites studied (antecubital fossa, volar forearm, and popliteal fossa; Figures S4 in Additional file 2; Table S5 in Additional file 1).

\section{Genus-level analysis}

In Tanner stage 1, Firmicutes (Streptococcus, Dolosigranulum, Gemella, and Granulicatella) and Proteobacteria 


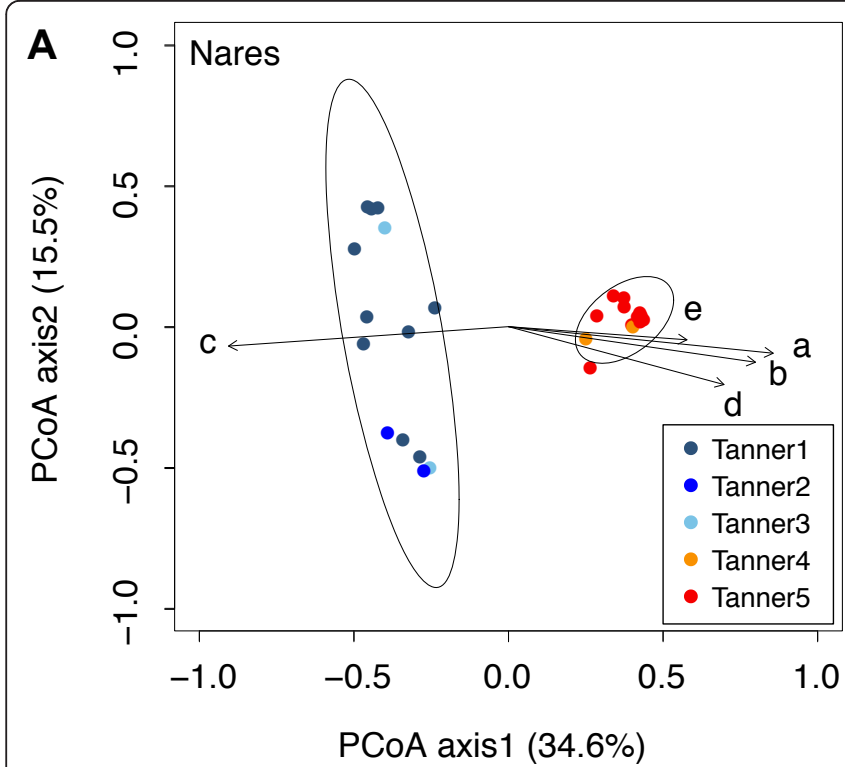

B
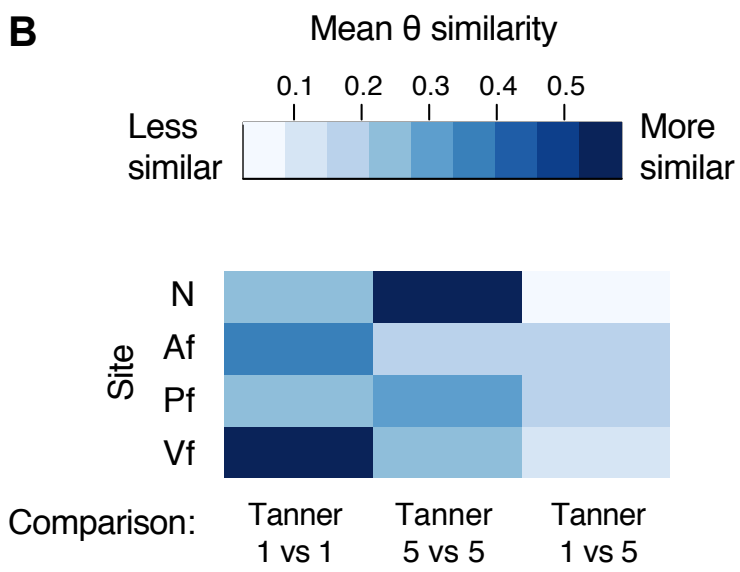

Figure 1 Microbial community-level statistics. (a) Nares samples clustered using principal coordinates analysis (PCoA) of the $\theta$ 'similarity' coefficient, which calculates similarity between two samples based on i) number of species in common between two samples and ii) their relative abundances. Colors represent different Tanner stages. Samples that have similar principal coordinates appear closer together, that is, are more similar. Ellipses represent the $95 \%$ confidence intervals based on standard deviation. Biplot arrows indicate the five most significant unique consensus taxonomies contributing to variation along axis 1. (Spearman correlations with axes and associated P-values are shown in Table $\mathbf{S 3}$ in Additional file 1). a, Propionibacterium; b, Corynebacterium; c, Streptococcus; d, Turicella; e, Anaerococcus. Additional skin sites are shown in Figure S2 in Additional file 2. (b) Heatmap of mean $\theta$ similarity coefficients of pairwise comparisons of communities within or between individuals of Tanner stage 1 and Tanner stage 5 (within-group, Tanner 1 versus Tanner 1 or Tanner 5 versus Tanner 5; between-group, Tanner 1 versus Tanner 5). Darker color indicates higher similarity: $\theta=0$ indicates that two samples have no species in common and $\theta=1$ indicates that two samples are identical. Site abbreviations: Af, antecubital fossa; N, nares; Pf, popliteal fossa; Vf, volar forearm.

(Moraxella, Haemophilus, and Neisseria) were the significantly overrepresented major genera in the nares (Table 1; Table S6 in Additional file 1). Actinobacteria (particularly Corynebacterium, Propionibacterium, and Turicella) were overrepresented in the nares of Tanner stage 5 individuals. Similar trends for many of these genera were observed for the three skin sites when comparing Tanner stages 1 versus 5, and 'Tanner1-3' versus 'Tanner4-5'.

\section{Species level analysis}

We further classified Streptococcus and Staphylococcus sequences by alignment to a curated reference database (Figure 3c; Figure S5 in Additional file 2; Tables S7 and S8 in Additional file 1). The commensal Streptococcus mitis/ Streptococcus pseudopneumoniae dominated streptococcal sequences overrepresented in children in all sampled sites, along with an overrepresentation of Streptococcus sanguinis and Streptococcus salivarius. Although overrepresentation of $S$. aureus and other staphylococci did not reach statistical significance in the Tanner 1 and Tanner 5 comparison due to high variability in relative abundance in the nares, $S$. aureus was significantly overrepresented in the 'Tanner1-3' nares compared with those of the older 'Tanner4-5' group.
Comparison with culture-based assessments and S. aureus as a reservoir of colonization

Given the potential of $S$. aureus as a pathobiont with the nares as a major reservoir for infection and persistence, each subject's nares sample was concurrently tested for presence and sensitivities of $S$. aureus via traditional culture-based techinques. Presence of $S$. aureus (see Materials and methods) was significantly associated with relative abundance of $S$. aureus nares genomic sequences $(\rho=$ $\left.0.73, P=9.1 \times 10^{-05}\right)$ across individuals of all Tanner stages, validating that relative abundance of genomic sequences is related to culture-based bacterial bioburden. $S$. aureus bioburden in the nares of Tanner 1 children was not significantly higher than in Tanner 5 adults (Figure 4; Table S10 in Additional file 1; Wilcoxon rank-sum test, $P$ $=0.41$ ). This was in contrast to the same comparison with sequence-based $S$. aureus relative abundances, with levels approaching significance $(P=0.11)$ for Tanner 1 versus Tanner 5, and reaching significance in the 'Tanner1-3' versus 'Tanner4-5' comparison $(P=0.05)$. These results suggest a trend of higher $S$. aureus relative abundance in younger children, with discordance with culture-based bioburden arising from either the higher resolution 


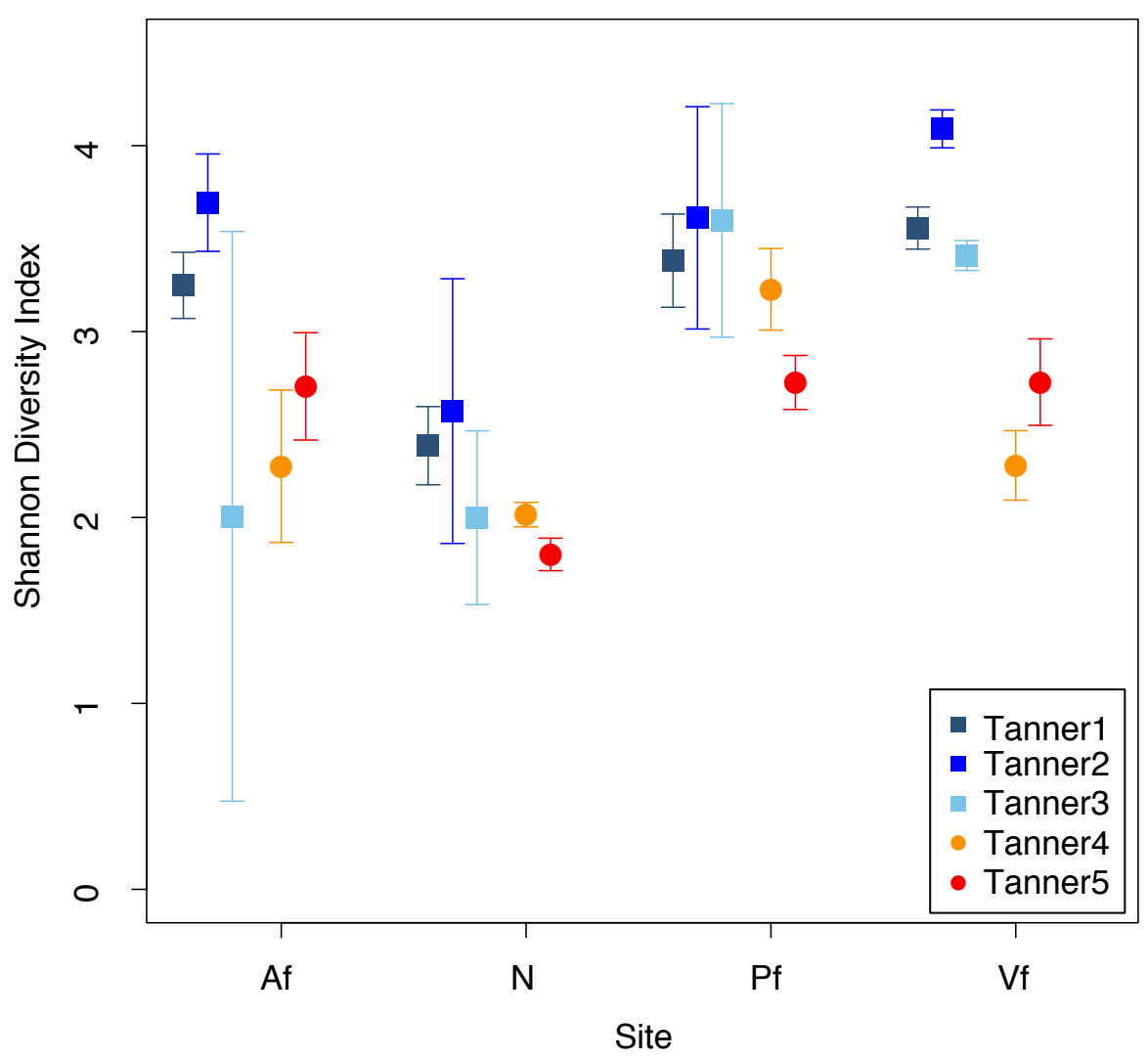

Figure 2 Mean Shannon diversity \pm SEM for each Tanner stage group (1-5) at all sites. Squares (blue hues) show data from Tanner stages 1 through 3; circles (red hues) indicate data from Tanner stages 4 and 5. Site abbreviations: Af, antecubital fossa; N, nares; Pf, popliteal fossa; Vf, volar forearm.

afforded by 16S-rRNA sequencing, or from the fact that bioburden is not necessarily an analogous reflection of relative community composition. None of the subjects sampled tested positive for methicillin-resistant S. aureus, suggesting that while $S$. aureus may be more abundant and occur more frequently in younger populations, there is no indication that carriage of methicillin resistance is dependent on age in our cohort.

Finally, to determine if the presence of $S$. aureus in the nares could serve as a reservoir for colonization to other skin sites, we determined if the incidence of $S$. aureus in the nares was associated with incidence in at least one of three distant skin sites. Across Tanner stages, we found a significant association between the incidence in the nares and incidence at other skin sites $\left(\mathrm{X}^{2}(1, N=26)=9.2\right.$, $P=0.002)$. We also determined that relative abundances of $S$. aureus in the nares and the distant skin sites were significantly correlated (Af, Spearman correlation $\rho=$ 0.53, $P=0.006$; Pf, $\rho=0.61, P=0.001$; Vf, $\rho=0.58$, $P=0.002)$. However, this trend was generally non-specific to Tanner 1 or $5\left(\mathrm{X}^{2}(1\right.$, Tanner $1 N=10$, Tanner $5 N=$ 10 for 'Tanner4-5') $=0.82,1.1 ; P=0.37,0.30$, respectively), and so we conclude that these data are consistent with the concept that the nares can serve as a reservoir for $S$. aureus, regardless of age.

\section{Discussion}

We identified a clear dichotomy in the skin and nares microbiomes between Tanner stage 1 children and Tanner stage 5 adults. Including a smaller number of subjects grouped as Tanner stages 2 to 4 , we observed that the microbial community memberships and structures of individuals of Tanner stages 2 and 3 trended towards more significantly resembling those of Tanner stage 1 than later stages, even though Tanner stages 2 and 3 are considered early stages of puberty. Physiologically, Tanner 4 individuals more closely resemble Tanner 5 individuals in terms of sexual maturity [13,14], sebum production [24], and the presence of other skin surface lipids in the face [25]. Correspondingly, we found that the microbial communities clustered into two distinct groups in which those of Tanner stages 1,2, and 3 differed significantly from those of Tanner stages 4 and 5 . Thus, we compared not only the microbiomes of Tanner 1 and Tanner 5 individuals, but also the microbiomes of two physiological groupings, young children ('Tanner1-3') and adolescent/post-adolescent 

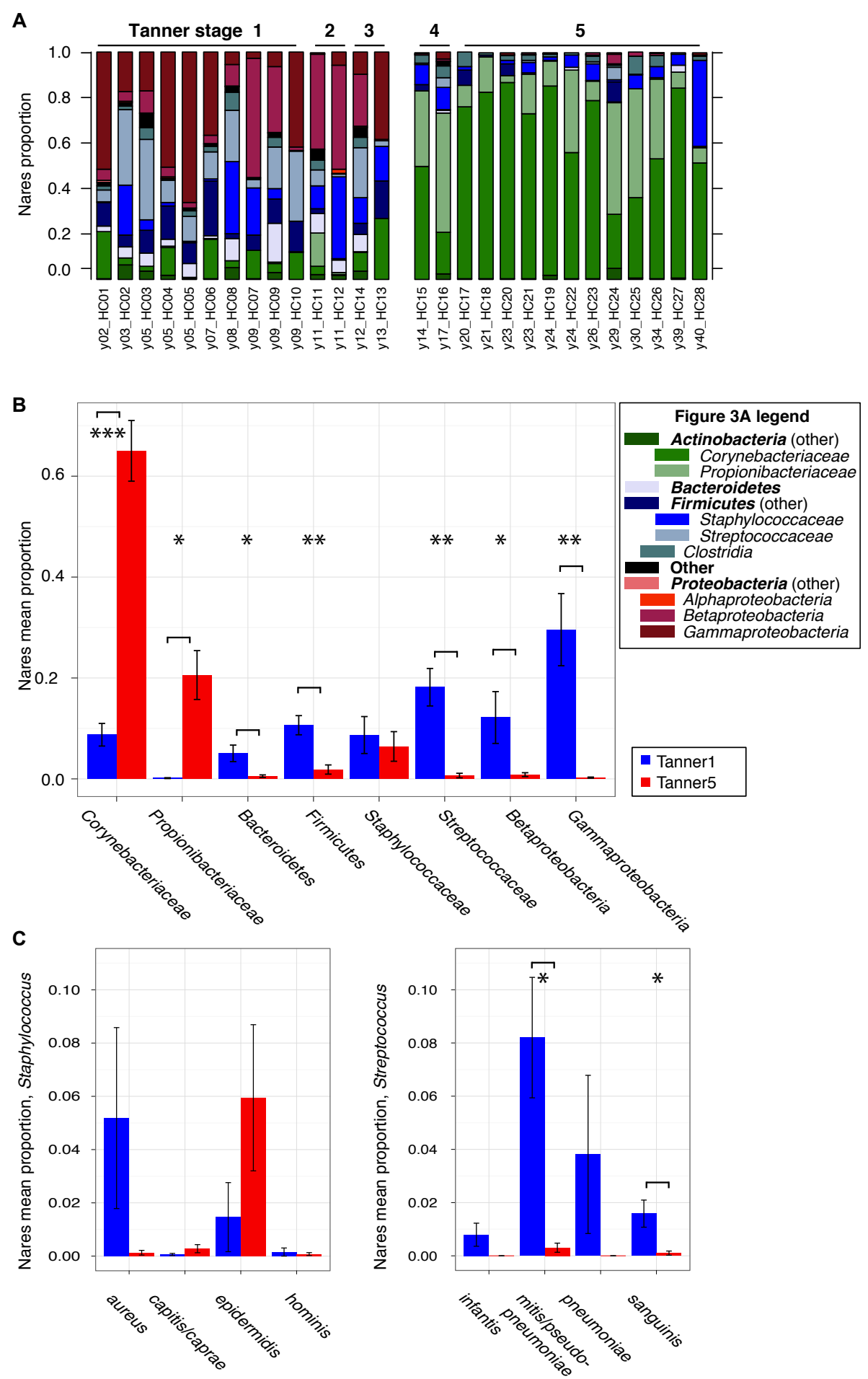

Figure 3 Bacterial taxonomic classifications and comparisons of relative abundance between Tanner stage groups. (a) Relative abundances of 12 major phyla-family taxonomies in nares. Subjects are ordered by age (' $\left.{ }^{* * \prime}\right)$ and Tanner staging is indicated. (b) Barplots of mean proportions of select major phyla-family classifications in nares. Statistical significance for false-discovery rate-adjusted $P$-values was assessed at $\alpha \leq 0.05 ;{ }^{*} P \leq 0.05,{ }^{*} P \leq 0.001,{ }^{* * * P} \leq 0.0001$. Tanner 1 means are shown in blue; Tanner 5 in red. The full version for the nares is provided in Figure S4 in Additional file 2. (c) Barplots of mean proportions of major species designations for genera Staphylococcus (left) and Streptococcus (right) in nares; descriptions are as in (b). 
Table 1 Abundant taxonomies differentially present in the nares

\begin{tabular}{|c|c|c|c|c|}
\hline Classification: phylum/class/family/genus ${ }^{\mathrm{a}}$ & Tanner 1 (mean $\pm \mathrm{SE})$ & * & Tanner 5 (mean $\pm \mathrm{SE})$ & Adjusted $P$-value \\
\hline Actinobacteria (other) & $2.1 \pm 0.7 \%$ & $\approx$ & $0.8 \pm 0.4 \%$ & $0.154^{b}$ \\
\hline Corynebacteriaceae & $8.8 \pm 2.2 \%$ & $<<$ & $65.0 \pm 6.0 \%$ & $1.18 \mathrm{E}-06$ \\
\hline Propionibacteriaceae & $0.2 \pm 0.1 \%$ & $<<$ & $20.6 \pm 4.8 \%$ & 0.0027 \\
\hline Corynebacterium & $8.7 \pm 2.2 \%$ & $<<$ & $49.6 \pm 5.6 \%$ & 0.0001 \\
\hline Propionibacterium & $0.1 \pm 0.1 \%$ & $<<$ & $20.6 \pm 4.8 \%$ & 0.0055 \\
\hline Rothia & $1.4 \pm 0.5 \%$ & $>$ & $0.1 \pm 0.1 \%$ & 0.0249 \\
\hline Turicella & $0.0 \pm 0.0 \%$ & $<<$ & $15.4 \pm 4.2 \%$ & 0.0119 \\
\hline Bacteroidetes (other) & $5.1 \pm 1.6 \%$ & $>>$ & $0.5 \pm 0.3 \%$ & 0.0158 \\
\hline Capnocytophaga & $0.7 \pm 0.2 \%$ & $>$ & $0.0 \pm 0.0 \%$ & 0.0007 \\
\hline Paraprevotella & $0.7 \pm 0.2 \%$ & $>$ & $0.0 \pm 0.0 \%$ & 0.0069 \\
\hline Porphyromonas & $1.4 \pm 0.5 \%$ & $>$ & $0.2 \pm 0.2 \%$ & 0.041 \\
\hline Prevotella & $0.6 \pm 0.2 \%$ & $>$ & $0.1 \pm 0.0 \%$ & 0.0201 \\
\hline Firmicutes (other) & $10.6 \pm 1.9 \%$ & $>>$ & $1.9 \pm 0.9 \%$ & 0.0009 \\
\hline Staphylococcaceae & $8.7 \pm 3.7 \%$ & $>$ & $6.4 \pm 2.9 \%$ & $0.6836^{\mathrm{b}}$ \\
\hline Streptococcaceae & $18.2 \pm 3.7 \%$ & $>>$ & $0.7 \pm 0.5 \%$ & 0.0003 \\
\hline Clostridia & $2.7 \pm 0.8 \%$ & $\approx$ & $2.8 \pm 0.7 \%$ & $0.9342^{b}$ \\
\hline Dolosigranulum & $8.6 \pm 2.2 \%$ & $>>$ & $1.7 \pm 0.9 \%$ & 0.0144 \\
\hline Gemella & $1.7 \pm 0.6 \%$ & $>$ & $0.0 \pm 0.0 \%$ & 0.0143 \\
\hline Granulicatella & $1.2 \pm 0.4 \%$ & $>$ & $0.0 \pm 0.0 \%$ & 0.0229 \\
\hline Streptococcus & $18.1 \pm 3.7 \%$ & $>>$ & $0.7 \pm 0.5 \%$ & 0.0006 \\
\hline \multicolumn{5}{|l|}{ Fusobacteria } \\
\hline Fusobacterium & $0.6 \pm 0.3 \%$ & $>$ & $0.0 \pm 0.0 \%$ & 0.0403 \\
\hline Proteobacteria (other) & $0.1 \pm 0.1 \%$ & $\approx$ & $0.0 \pm 0.0 \%$ & $0.3356^{b}$ \\
\hline Alphaproteobacteria & $0.3 \pm 0.1 \%$ & $>$ & $0.1 \pm 0.0 \%$ & 0.0317 \\
\hline Betaproteobacteria & $12.2 \pm 5.1 \%$ & $>>$ & $0.8 \pm 0.4 \%$ & 0.0365 \\
\hline Gammaproteobacteria & $29.6 \pm 7.2 \%$ & $>>$ & $0.3 \pm 0.1 \%$ & 0.0009 \\
\hline Neisseria & $2.3 \pm 0.9 \%$ & $>$ & $0.1 \pm 0.1 \%$ & 0.0201 \\
\hline Haemophilus & $3.7 \pm 1.5 \%$ & $>$ & $0.0 \pm 0.0 \%$ & 0.0256 \\
\hline Moraxella & $24.1 \pm 7.0 \%$ & $>>$ & $0.0 \pm 0.0 \%$ & 0.0055 \\
\hline
\end{tabular}

${ }^{a}$ Represents proportion in the microbiome as a whole, not a subset of the taxonomy. Higher taxonomic levels are listed first. ${ }^{b}$ Not significant; see Additional file 2 for additional details. *Greater or less than; double signs indicate a difference of notable magnitude.

individuals ('Tanner4-5'). We observed that a greater diversity of bacteria, including Streptococcus and Gramnegatives Moraxella, Haemophilus, and Neisseria, dominated the microbiomes of Tanner stage 1 children. In contrast, Tanner stage 5 individuals had few to none of these taxa; their microbiomes were dominated instead by lipophilic bacteria, including Propionibacterium, Corynebacterium, and Turicella, that are associated with sebaceous skin regions [26,27]. The results comparing Tanner stages 1 to 3 versus stages 4 to 5 were highly concordant.

We attribute the prevalence of lipophilic taxa to increased epidermal lipids resulting from increased hormone-stimulated sebaceous gland activity during puberty. Higher sebum concentration markedly favors colonization by lipophilic bacteria at the expense of other flora - metabolism of skin lipids reduces skin $\mathrm{pH}$, which in turn inhibits staphylococcal and streptococcal species $[28,29]$. Other skin changes that occur during puberty include increased density and thickness of body hair and increased apocrine gland activity, which may also contribute to the observed changes in microbial communities. Alternatively, subsets within the larger immune system, possibily immune components in skin, may be less mature in younger individuals and may permit the colonization and growth of a wider range of bacteria, consistent with the higher community-wide diversity we observed in younger children.

Samples from characteristially sebaceous sites, such as the head or upper torso, have the highest density of sebaceous glands and have been shown to have a different microbiome from less sebaceous sites [2]. The skin sites included in this study do not typically have the highest density of sebaceous glands. However, the antecubital/popliteal fossa and volar forearms contain hair follicles that develop sebaceous glands during puberty, but to a lesser degree than skin sites classically defined as sebaceous. It is possible, as suggested in our study, that even moderate changes in lipids on the skin may be sufficient to drive colonization of lipophilic bacteria in the skin. 


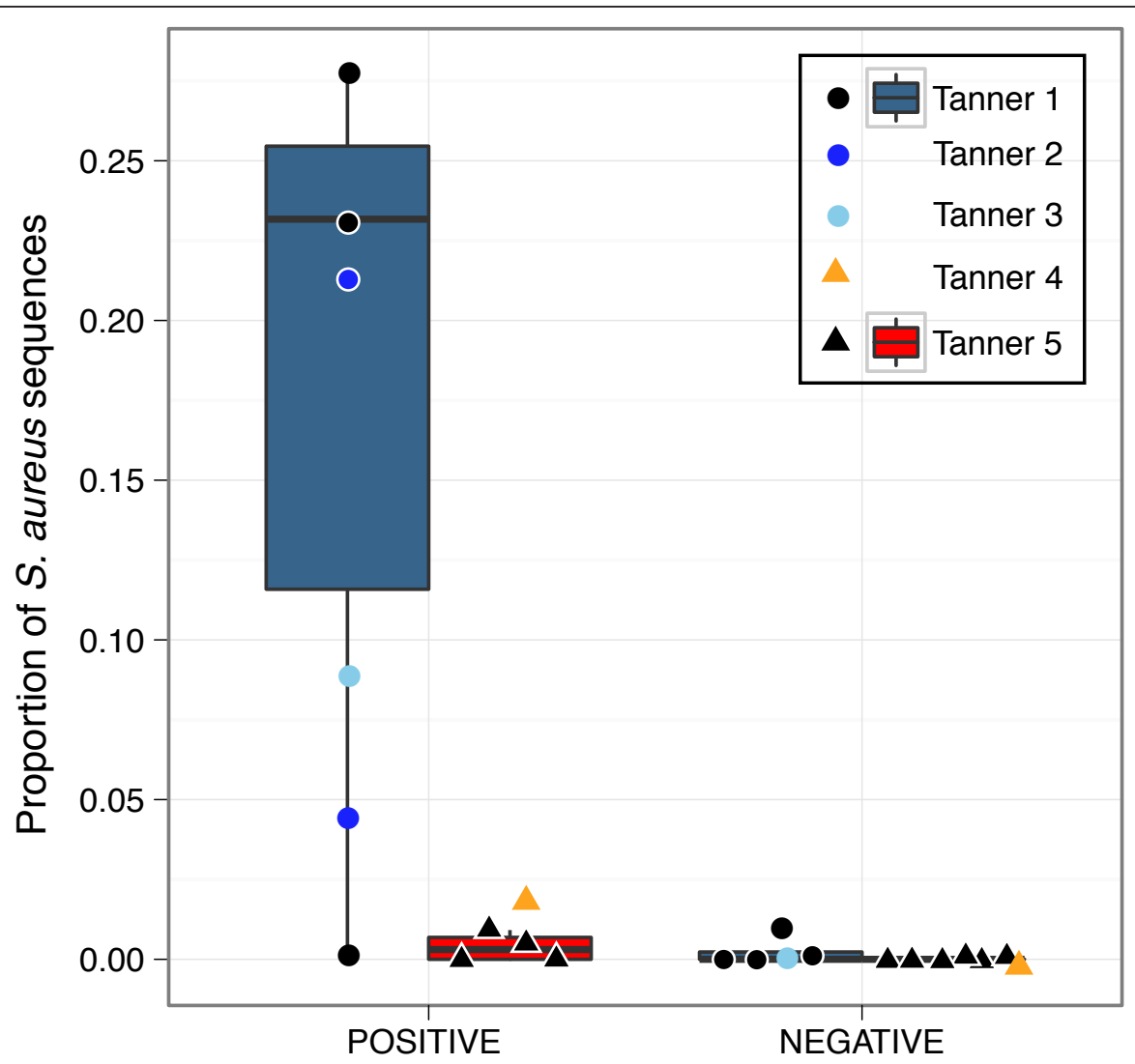

Results of $S$. aureus culture

Figure 4 Comparison between S. aureus culture and 16S-rRNA sequencing. Cultures were scored based on the following criteria: zero colonies of S. aureus (negative for S. aureus) and $\geq 1$ colony (positive for S. aureus). The boxplot represents the nares culture results for $S$. aureus compared to the sequence-based relative abundance values of $S$. aureus. Tanner 1 group in gray-blue; Tanner 5 in red. Points for additional Tanner stages are plotted but do not contribute to the boxplot dimensions.

We did not observe significant gender-related differences associated with numerous sexually dimorphic traits that occur during puberty. For example, production and metabolism of sex hormones cause differential production and composition of sebum between males and females $[24,30]$. Again, even modest increases in lipid production may be sufficient to alter microbiome colonization and composition. Alternatively, previous studies characterizing sebum production have focused on the forehead, a sebaceous site where the density of sebaceous glands can outnumber those at other body sites by over 10-fold [30]. Moist and dry skin sites, such as those surveyed in our study, may thereby exhibit less sexual dimorphism. Another possibility is that there may be differences in the absolute abundances of bacteria, as there have been reports of higher bacterial loads on male adult skin compared with that of females [31], but the relative compositions of the microbiota are similar between gender.

While all sites surveyed showed distinct maturationrelated microbial differences, this contrast was particularly striking in the nares, which can serve as a reservoir for potential pathogens, including S. aureus and S. pneumoniae [23]. Studying S. aureus in the context of its microbial communities will facilitate understanding how microbial communities contribute to the initiation and perpetuation of skin disease. For example, AD is a primarily pediatric disease that often resolves by adolescence and adulthood. We observed an elevated incidence and abundance of $S$. aureus in the nares of the 'Tanner1-3' group, which may be a factor in the higher incidence, recurrence, and severity of $\mathrm{AD}$ observed in this age group. Our findings do not necessarily explain the resolution of $\mathrm{AD}$, particularly in those patients whose symptoms improve before puberty. However, $\mathrm{AD}$ is a complex inflammatory disease, and human-associated microbial communities may be a contributory factor.

Moreover, our results demonstrate that $S$. aureus abundance and incidence in the nares was significantly associated with those found at distant skin sites. Of note, this study cannot assess the directionality of colonization. 
However, staphylococcal and streptococcal species in the nares are replaced by lipophilic and other bacteria during late/post-adolescence - a potential contributor to the age-related reduction in incidence and severity of skin disorders, such as AD. Lipophiles, once established in the skin and nares of mature individuals, may create an inhospitable environment for staphylococci, thus reducing both prevalence as well as bioburden of $\mathrm{AD}$ and $S$. aureus. These findings raise an interesting evolutionary question regarding host-microbiome interactions that may reduce the ability to harbor potential pathogens upon reaching reproductive age.

In all Tanner stages examined, the skin sites in these healthy subjects were significantly more similar to each other as compared to the nares. The relative similarity of skin sites is consistent with prior studies that showed that samples clustered based on the body sites of gut, skin, vagina, and oral cavity $[3,32]$. These findings are in contrast to patients with atopic dermatitis, who demonstrate a distinct difference in sites more characteristically affected with skin disease, such as the antecubital fossa compared to the volar forearm [12].

The significant shift in elevated relative abundances of Corynebacterium, Propionibacterium, and other lipophiles in the nares and increased prevalence in the flexural sites (antecubital, popliteal fossa) and even non-flexural sites (volar forearm) with sexual maturation was unexpected. Maturation of sebaceous glands and sebum secretion in early childhood may progress asynchronously across body sites, occurring earliest at typically sebaceous and proximal skin regions, including the face, back, and chest. Transition into a more sexually mature microbiome at distal and less characteristically sebaceous regions may be delayed. The delayed maturation at these sites may contribute to the persistence into adolescence of diseases that are linked to staphylococcal species, given the antagonism of lipophilic species and staphylococci $[28,29]$.

While our results generally corroborate lower-resolution culture-based methods showing increased diversity in the skin flora of children and increased colonization by diptheroids at other skin sites in adults $[4,5]$, genomicsbased methods offer more comprehensive detection and quantification of both cultivatable and fastidious organisms. Results from the culture-based methods may be skewed towards fast-growing organisms and are most useful for incidence-based studies as they give less information on the relative abundance of a taxa in the microbial community as a whole. For example, we observed trace, if any, amounts of Sarcina in either children or adults as reported by culture-based studies [4,5], and Neisseria, which we observed to be significantly overrepresented in the 'Tanner1-3' group, occurred in 'high incidence' in adults as well as children. A likely explanation for this discrepancy is that these studies tested different body sites, such as the forehead, toewebs, hands, and ear. Previous work from our laboratory and others has demonstrated great taxonomic diversity between different body sites $[2,3]$, despite having similar physiological characteristics like sebaceous, moist, or dry microenvironments. These culture-based studies also classified diptheroids as 'highincident' in both children and adults, which we corroborate; however, we show that their representation in the microbial communities is significantly diminished in younger indiviuduals. Finally, our study contrasts with previous work examining the normal flora of skin in different age groups in that we focused on sites with clinical predilection to diseases such as $\mathrm{AD}$, such as the antecubital and popliteal fossae, in which these dynamics between liphophiles and potential pathogenic genera such as Staphylococcus and Streptococcus are of particular clinical interest and may be exploited for preventative or therapeutic (pre/ probiotic) approaches.

\section{Conclusions}

Future studies will further define microbial interactions, disease-causing perturbations to microbial ecology, and host-commensal microbe relationships. Moreover, therapies based on probiotics or altering the balance of microbial communities have the potential to augment or bypass antibiotic use, decreasing the rise of antibiotic-resistant bacteria. Our results from this genomic microbial survey demonstrate a significant difference in the microbiomes of children versus adults at body sites relevant to infections and inflammatory skin diseases. These microbiome differences must be considered in the design and interpretation of studies concerning the etiology, prevention, and treatment of skin diseases with microbial etiology or influence.

\section{Additional material}

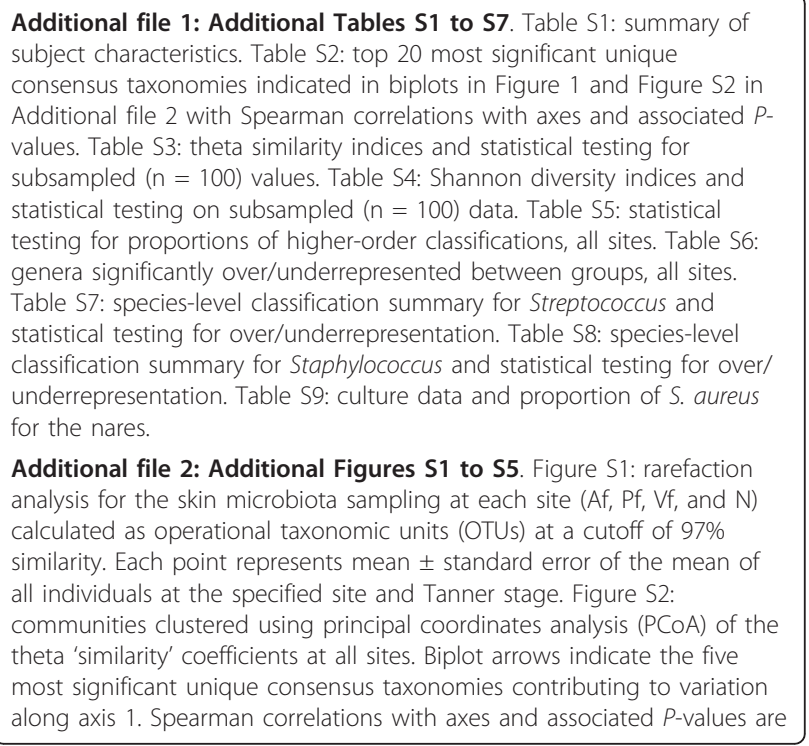

Additional file 1: Additional Tables S1 to S7. Table S1: summary of subject characteristics. Table S2: top 20 most significant unique consensus taxonomies indicated in biplots in Figure 1 and Figure S2 in Additional file 2 with Spearman correlations with axes and associated $P$ values. Table S3: theta similarity indices and statistical testing for subsampled $(n=100)$ values. Table S4: Shannon diversity indices and statistical testing on subsampled $(n=100)$ data. Table S5: statistical testing for proportions of higher-order classifications, all sites. Table S6: genera significantly over/underrepresented between groups, all sites. Table S7: species-level classification summary for Streptococcus and statistical testing for over/underrepresentation. Table S8: species-level classification summary for Staphylococcus and statistical testing for over/ underrepresentation. Table S9: culture data and proportion of S. aureus for the nares.

Additional file 2: Additional Figures S1 to S5. Figure S1: rarefaction analysis for the skin microbiota sampling at each site (Af, Pf, Vf, and N) calculated as operational taxonomic units (OTUs) at a cutoff of $97 \%$ similarity. Each point represents mean \pm standard error of the mean of all individuals at the specified site and Tanner stage. Figure S2: communities clustered using principal coordinates analysis ( $\mathrm{PCOA}$ ) of the theta 'similarity' coefficients at all sites. Biplot arrows indicate the five most significant unique consensus taxonomies contributing to variation along axis 1. Spearman correlations with axes and associated $P$-values are 
shown in Table S3 in Additional file 1. Sites indicated are: Af, antecubital fossa; Pf, popliteal fossa; $\mathrm{Vf}$, volar forearm. The nares plot is shown in Figure 1a. Analysis of molecular variance (AMOVA) testing differences in centroids is indicated for both Tanner 1 versus Tanner 5 and Tanner $1-3^{\prime}$ versus 'Tanner4-5'. Figure S3: bacterial taxonomic classifications for additional sites. Tanner stage is indicated. Figure S4: mean relative abundances by Tanner group for 12 major phyla-family taxonomic classifications. ${ }^{*} P \leq 0.05,{ }^{* *} P \leq 0.001,{ }^{* * *} P \leq 0.0001$. Tanner 1 in red; Tanner 5 in blue. Figure S5: mean relative abundances by Tanner group for major species designations for genera Streptococcus and Staphylococcus. ${ }^{*} P \leq 0.05,{ }^{* *} P \leq 0.001,{ }^{* * *} P \leq 0.0001$. Tanner 1 in red; Tanner 5 in blue.

\section{Abbreviations}

AD: atopic dermatitis; Af: antecubital fossa; AMOVA: analysis of molecular variance; ANOVA: analysis of variance; N: nares/nostril; OTU: operational taxonomic unit; PCoA: principal coordinates analysis; Pf: popliteal fossa; Vf: volar forearm

\section{Acknowledgements}

We thank Clay Deming, Effie Nomicos, and Deborah Schoenfeld for their underlying efforts; and Maria L Turner, Isaac Brownell, and Mark C Udey for helpful discussions. This work was supported by NIH NHGRI and NCI Intramural Research Programs and in part by 1K99AR059222 (HHK). Sequencing was funded by grants from the National Institutes of Health (1UH2AR057504-01 and 4UH3AR057504-02).

\section{Author details}

1 Genetics and Molecular Biology Branch, National Human Genome Research Institute, NIH, 49 Convent Dr., Bethesda, MD 20814, USA. ${ }^{2}$ Biometric Research Branch, Division of Cancer Treatment and Diagnosis, National Cancer Institute, NIH, 6130 Executive Blvd, Rockville, MD 20852, USA. ${ }^{3}$ Dermatology Branch, Center for Cancer Research, National Cancer Institute, NIH, 10 Center Dr., Bethesda, MD 20814, USA.

\section{Authors' contributions}

Study concept and design: JO, JAS, HHK. Acquisition of data: JO, HHK. Drafting of the manuscript: JO, JAS, HHK. Critical revision of the manuscript for important intellectual content: SC, ECP, JAS, HHK. Statistical analysis: JO, ECP. Administrative, technical, or material support: SC, ECP, JAS, HHK. Study supervision: JAS, HHK. All authors read and approved the manuscript.

\section{Competing interests}

The authors declare that they have no competing interests.

Received: 23 May 2012 Revised: 17 August 2012

Accepted: 10 October 2012 Published: 10 October 2012

\section{References}

1. Littman DR, Pamer EG: Role of the commensal microbiota in normal and pathogenic host immune responses. Cell Host Microbe 2011, 10:311-323.

2. Grice EA, Kong HH, Conlan S, Deming CB, Davis J, Young AC, NISC Comparative Sequencing Program, Bouffard GG, Blakesley RW, Murray PR, Green ED, Turner ML, Segre JA: Topographical and temporal diversity of the human skin microbiome. Science 2009, 324:1190-1192.

3. Costello EK, Lauber CL, Hamady M, Fierer N, Gordon II, Knight R: Bacterial community variation in human body habitats across space and time. Science 2009, 326:1694-1697.

4. Somerville DA: The normal flora of the skin in different age groups. $\mathrm{Br}$ J Dermatol 1969, 81:1365-2133.

5. Somerville DA: The effect of age on the normal bacterial flora of the skin. Br J Dermatol 1969, 81:1365-2133.

6. Maier RM, Pepper IL, Gerba CP: Environmental Microbiology. 2 edition. Academic Press; 2009.

7. Dominguez-Bello MG, Costello EK, Contreras M, Magris M, Hidalgo G, Fierer N, Knight R: Delivery mode shapes the acquisition and structure of the initial microbiota across multiple body habitats in newborns. Proc Natl Acad Sci U S A 2010, 107:11971-11975.
8. Capone KA, Dowd SE, Stamatas GN, Nikolovski J: Diversity of the human skin microbiome early in life. J Invest Dermatol 2011, 131:2026-2032.

9. Largo RH, Prader A: Pubertal development in Swiss girls. Helv Paediatr Acta 1983, 38:229-243.

10. Largo RH, Prader A: Pubertal development in Swiss boys. Helv Paediatr Acta 1983, 38:211-228.

11. Asher M, Keil U, Anderson H, Beasley R, Crane J, Martinez F, Mitchell E, Pearce N, Sibbald B, Stewart AW, Weiland SK, Williams HC: International Study of Asthma and Allergies in Childhood (ISAAC): rationale and methods. Eur Resp J 1995, 8:483-491.

12. Kong HH, Oh J, Deming CB, Conlan S, Grice EA, Beatson MA, Nomicos E, Polley E, Komarow HD, NISC Comparative Sequencing Program, Murray PR, Turner ML, Segre JA: Temporal shifts in the skin microbiome associated with disease flares and treatment in children with atopic dermatitis. Genome Res 2012, 22:850-859.

13. Marshall WA, Tanner JM: Variations in the pattern of pubertal changes in boys. Arch Dis Child 1970, 45:13-23.

14. Marshall WA, Tanner JM: Variations in pattern of pubertal changes in girls. Arch Dis Child 1969, 44:291-303.

15. Schloss PD, Westcott SL, Ryabin T, Hall JR, Hartmann M, Hollister EB, Lesniewski RA, Oakley BB, Parks DH, Robinson CJ, Sahl JW, Stres B, Thallinger GG, Van Horn DJ, Weber CF: Introducing mothur: open-source, platform-independent, community-supported software for describing and comparing microbial communities. Appl Environ Microbiol 2009, 75:7537-7541.

16. DeSantis TZ, Hugenholtz P, Larsen N, Rojas M, Brodie EL, Keller K, Huber T, Dalevi D, Hu P, Andersen GL: Greengenes, a chimera-checked 16S rRNA gene database and workbench compatible with ARB. Appl Environ Microbiol 2006, 72:5069-5072.

17. DeSantis TZ, Hugenholtz P, Keller K, Brodie EL, Larsen N, Piceno YM, Phan R, Andersen GL: NAST: a multiple sequence alignment server for comparative analysis of 165 rRNA genes. Nucleic Acids Res 2006, 34: W394-W399.

18. Edgar RC, Haas BJ, Clemente JC, Quince C, Knight R: UCHIME improves sensitivity and speed of chimera detection. Bioinformatics 2011, 27:2194-2200.

19. Wang Q, Garrity GM, Tiedje JM, Cole JR: Naive Bayesian classifier for rapid assignment of rRNA sequences into the new bacterial taxonomy. Appl Environ Microbiol 2007, 73:5261-5267.

20. Sheneman L, Evans J, Foster JA: Clearcut: a fast implementation of relaxed neighbor joining. Bioinformatics 2006, 22:2823-2824.

21. Benjamini $Y$, Hochberg $Y$ : Controlling the false discovery rate: a practical and powerful approach to multiple testing. J R Stat Soc B (Methodological) 1995, 57:289-300.

22. Excoffier L, Smouse PE, Quattro JM: Analysis of molecular variance inferred from metric distances among DNA haplotypes: application to human mitochondrial DNA restriction data. Genetics 1992, 131:479-491.

23. von Eiff C, Becker K, Machka K, Stammer H, Peters G: Nasal carriage as a source of Staphylococcus aureus bacteremia. N Engl J Med 2001, 344:11-16.

24. Cotterill JA, Cunliffe WJ, Williamson B, Bulusu L: Age and sex variation in skin surface lipid composition and sebum excretion rate. Br J Dermatol 1972, 87:333-340.

25. Ramasastry P, Downing DT, Pochi PE, Strauss JS: Chemical composition of human skin surface lipids from birth to puberty. I Investigative Dermatol 1970, 54:139-144.

26. Roth RR, James WD: Microbial ecology of the skin. Annu Rev Microbiol $1988,42: 441-464$.

27. McGinley KJ, Webster GF, Leyden JJ: Regional variations of cutaneous propionibacteria. Appl Environ Microbiol 1978, 35:62-66.

28. Gribbon EM, Cunliffe WJ, Holland KT: Interaction of Propionibacterium acnes with skin lipids in vitro. J General Microbiol 1993, 139:1745-1751.

29. Hentges DJ: The anaerobic microflora of the human body. Clin Infect Dis 1993, 16:S175-S180.

30. Giacomoni PU, Mammone T, Teri M: Gender-linked differences in human skin. J Dermatol Sci 2009, 55:144-149.

31. Marples RR: Sex, constancy, and skin bacteria. Arch Dermatol Res 1982, 272:317-320.

32. Consortium THMP: Structure, function and diversity of the healthy human microbiome. Nature 2012, 486:207-214.

\section{doi:10.1186/gm378}

Cite this article as: Oh et al: Shifts in human skin and nares microbiota of healthy children and adults. Genome Medicine 2012 4:77. 